\title{
RADII AND FLUXES OF LONG-PERIOD CEPHEIDS
}

\author{
A. OPOLSKI \\ Wroclaw Astronomical Observatory, Wroclaw, Poland
}

\begin{abstract}
The radii of 9 long-period Cepheids have been determined by means of the modificated Wesselink method and some general conclusions have been formulated. The relations $R-P$ and $F_{v}-M_{v}$ for stars recognized as fundamental and first overtone pulsators have been established.
\end{abstract}

The determination of stellar fluxes $F_{v}$ in the $V$ system, relating to the solar flux $F_{v}$ may be based on the fundamental relation:

$$
\log F_{v} / F_{v \odot}=-0.4\left(M_{v}-M_{v \odot}\right)-2 \log R / R_{\odot}
$$

In order to use this relation for long-period Cepheids we should secure the correct values of their radii. In this case they are more essential than the absolute magnitudes $M_{v}$ because of the coefficients 0.4 and 2 respectively. The radii of pulsating stars are usually computed with the well known Wesselink method, which has been applied in our considerations but with some modifications. The proposed alterations should facilitate the practical use of this method and should increase the accuracy of the results.

In the Wesselink method we try to separate two factors acting simultaneously and defining the observed changes of star magnitude: the changes of fluxes and of radii

$$
\Delta V=-5 \log (1+\Delta R / R)-2.5 \Delta \log F_{v},
$$

where $\Delta R$ is the displacement resulting from the integration of the radial velocities. In practice the component $5 \log (1+\Delta R / R)$ is most frequently replaced by a more convenient but only approximatively equivalent value $2.17(\Delta R / R)$.

But we may proceed in the following way. Let us fix that $R_{\max }$ denotes the maximum of the radius, which for the Cepheids occur near the phases $0.3-0.4$ and is rather flat. Now we introduce the correcting factor $c$ in order to get the exact equality

$$
5 \log \left(1+\Delta R / R_{\max }\right)=2.17\left(\Delta R / R_{\max }\right) \cdot c .
$$

The value $c$ depends in small degree on $\Delta R / R_{\max }$ :

\begin{tabular}{ll}
\hline$\Delta R / R_{\max }$ & $c$ \\
\hline 0.00 & 1.000 \\
-0.05 & 1.028 \\
-0.10 & 1.055 \\
-0.15 & 1.084 \\
-0.20 & 1.116 \\
\hline
\end{tabular}

and can be computed even by applying an approximate value of $R_{\max }$. The Equation 
(3) can be transformed as:

$$
5 \log \left(1+\Delta R / R_{\max }\right)=N r,
$$

where $N=2.17 / R_{\max }$ determines the maximum value of the radius, and the 'rectified displacement' $r$ :

$$
r=\Delta R \cdot c
$$

assures the exact proportionality to the photometric effects expressed in the magnitude scale.

By this method we have for each phase three values determining the state of a star: $V,(B-V)$, and $r$. The farther procedure can be continued in two ways: graphically or numerically.

\section{Graphical Method}

According to Wesselink assumption for two phases with equal $(B-V)$ or for $\Delta(B-V)=0$, we have also the same values of $F_{v}$ or $\Delta \log F_{v}=0$. So the Equation (2) is reduced to

$$
\begin{aligned}
& \Delta V=-N \Delta r \\
& \text { or } \quad N=-\Delta V / \Delta r ; \quad \text { or } \quad R_{\max }=-2.17(\Delta r / \Delta V) .
\end{aligned}
$$

Let us draw two graphs with the common axis $(B-V)$, Figure 1. Perpendicularly are $V$ axis upwards and $r$ axis downwards. By means of the values $V,(B-V)$, and $r$

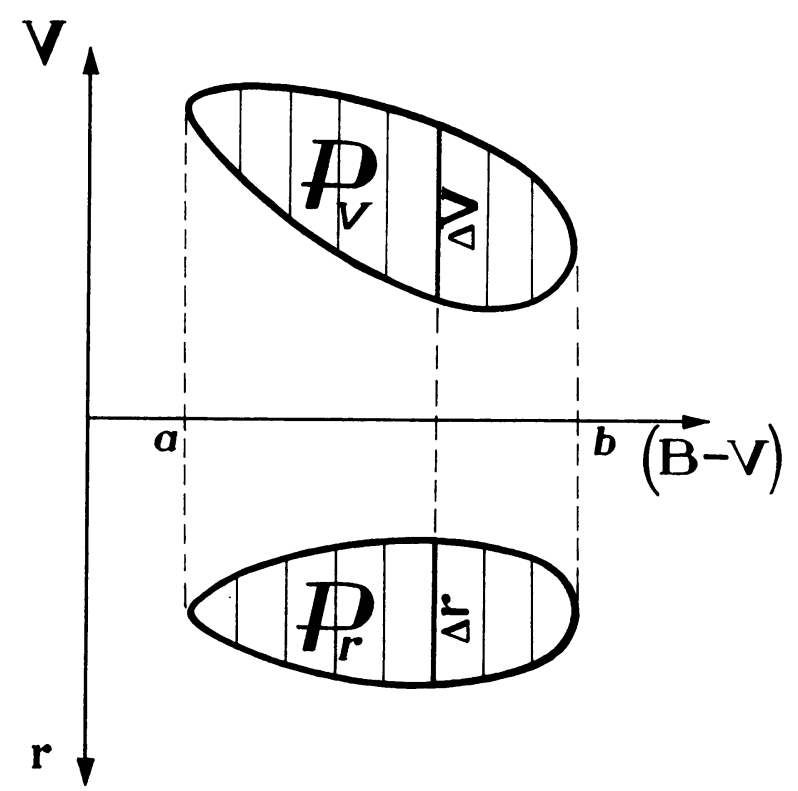

Fig. 1. Graphical method of $N$ and $R_{\max }$ determination. 
we trace two loops described by star during one period. Each line perpendicular to the $(B-V)$ axis and cutting both loops passes through the points with equal $(B-V)$. So the sections of the loops $\Delta V$ and $\Delta r$ can be used as the values needed in the formula (6). But each such a cutting has this property. So it follows that $N$ can be calculated as

$$
N=-\frac{\int_{a}^{b} \Delta V \mathrm{~d}(B-V)}{\int_{a}^{b} \Delta r \mathrm{~d}(B-V)},
$$

where the integration includes the whole range of $(B-V)$ changes. In practice this integration can simply be executed by measuring the surfaces of the loops $P_{v}$ and $P_{r}$.

$$
N=P_{v} / P_{r} ; \quad R_{\max }=2.17 P_{r} / P_{v}
$$

\section{Numerical Method}

In this method we change the Wesselink basing assumption and enlarge the range of its applicability by introducing the coefficient $k$ so that

$$
-2.5 \Delta \log F_{v}=k \Delta(B-V) \text {. }
$$

The coefficient $k$ is regarded as a constant or as a mean value in the case, when it depends on the phases used in the formula (9). In terms of fluxes the Wesselink assumption: $\Delta \log F_{v}=0$, when $\Delta(B-V)=0$ is equivalent to the statement that $F_{v}$ is a monotonic function of $F_{B}$, but not of the shape $F_{v}=A F_{B}$, with constant $A$. In this last case we would have constant $(B-V)$ and it would not be possible to separate photometrically the changes of fluxes from the changes of radius. Whereas according to the assumption (9) we have

$$
F_{v}=A F_{B}^{a} ; \quad a \neq 1 ; \quad k=\frac{a}{1-a} .
$$

This formula with two parameters $A$ and a can represent, at least in approximation, a rather large class of real relations between both kinds of fluxes in the range of changes occuring during the star pulsation. Though it is to be remarked that in reality the fluxes as the functions of many atmospheric parameters - model, temperature, gravity cannot be connected by only one function during the whole period.

By means of the formulas (4) and (9) the Equation (2) gains the simple shape:

$$
\Delta V=-N \Delta r+k \Delta(B-V) \text {. }
$$

This type of relation was used by Opolski and Krawiecka (1956) and by Latyshev (1964). The differences $\Delta V, \Delta r$, and $\Delta(B-V)$ can be calculated for each free chosen pair of phases. But in order to decrease the influence of the observational errors, it seems to be advantageous to calculate first the mean values $\bar{V}, \bar{r}$ and $(\overline{B-V})$, and 
then to use the differences between the individual values and their mean: $\Delta V=V-\bar{V}$; $\Delta r=r-\bar{r} ; \Delta(B-V)=(B-V)-\overline{(B-V)}$. So we can form the system of the Equations (11) with two unknown $N$ and $k$. Taking into consideration the fact that $\Delta(B-V)$ are less accurate than $\Delta V$, we'can solve this system in the form

$$
(B-V)=\frac{1}{k} V+\frac{N}{k} \Delta_{r} .
$$

The individual values $V, B-V$ and $r$ should be suitably distributed on the light-, colour- and displacement-curves. By this method we can get for each Cepheid two characteristic quantities: $N$-defining the radius of the star and $k$ - which is a measure of the relation between the fluxes $F_{v}$ and $F_{B}$. Also the accuracy of the values $N$ and $k$ can be calculated by standard methods.

We remark still, that the coefficient $k$ may be obtained separately by the graphical method analogically to the $N$ determination. For two phases with $\Delta r=0$ we have $\Delta V=k \Delta(B-V)$ and the surface of two loops on the planes in the coordinate systems $V, r$ and $(B-V), r$ determine the coefficient $k$.

As an example of the application of the above described methods we present in Table I the results obtained for 9 Cepheids. General remarks which can be deduced from these results are as follows:

(1) There are no systematic differences between $N$ values obtained by graphical and numerical methods. But in Table I the results received by numerical methods are given.

TABLE I

Radii and fluxes of Cepheids

\begin{tabular}{|c|c|c|c|c|c|c|c|}
\hline Star & $\log P$ & $\left\langle M_{V}\right\rangle_{\text {int }}$ & $\mathrm{Sp}$ & $\log R_{\max }$ & $\log R$ & $\log _{F_{v_{\odot}}} \frac{F_{v}}{F}$ & $\left(\begin{array}{ll}\log & F_{v} \\
& F_{v_{\odot}}\end{array}\right)$ \\
\hline$\eta$ Aql & 0.855 & -3.9 & F 8.8 & 7.68 & 7.66 & -0.22 & -0.18 \\
\hline W Sgr & 0.880 & -4.0 & F 7.7 & 7.69 & 7.67 & -0.19 & -0.21 \\
\hline S Sge & 0.923 & -4.1 & F 8.7 & 7.75 & 7.73 & -0.26 & -0.22 \\
\hline$\beta$ Dor & 0.993 & -4.3 & F 8.1 & 7.79 & 7.77 & -0.27 & -0.27 \\
\hline$\zeta$ Gem & 1.007 & -4.3 & F 9.0 & 7.79 & 7.78 & -0.27 & -0.22 \\
\hline X Cyg & 1.215 & -4.8 & G 0.0 & 8.03 & 8.00 & -0.56 & -0.46 \\
\hline T Mon & 1.432 & -5.5 & G 1.1 & 8.14 & 8.10 & -0.50 & -0.34 \\
\hline $1 \mathrm{Car}$ & 1.551 & -5.9 & F 9.1 & 8.18 & 8.15 & -0.42 & -0.36 \\
\hline SV Vul & 1.655 & -6.1 & G 2.1 & 8.40 & 8.36 & -0.77 & -0.57 \\
\hline
\end{tabular}

(2) When we try to improve the accuracy of $N$ and $k$ by shifting the phases of the 'rectified displacement' $r$, as it was suggested by Fernie and Hube (1967), it appears that the smallest errors of $N$ and $k$ occures at the diminishing of the phases $r$ (and $\Delta R$ ) by about $0^{P} .05$. This is particularly essential near the phases of sharp minimum of the radius. From the photometric data we should fix $R_{\min }$ in the phases near 0.87 , whereas from the radial velocities $R_{\min }$ appears later, at the phases 0.92 . This can be explained as due to the fact that, after common falling down of the whole atmosphere, the 
movement upwards begins first in the photospheric layer, responsible for the radiation measured in the $B$ and $V$ systems, and then $0^{\text {P. }} .05$ later, in the upper layers of the atmosphere, where the narrow absorption lines used for radial velocity determination are formed. This may be connected with the emission effects observed in some lines at these phases. Similar conclusion is to be found in the paper by Latyshev (1964).

(3) The best accuracy of the results gained by suitable diminishing of $r$ phases is joint with the smallest value of $N$ and consequently with the greatest value of $R_{\max }$. The mean values $\bar{R}=R_{\max }-\left(\frac{1}{2}\right.$ amplitude of $\left.\Delta R\right)$ given in Table I, for long-period Cepheids are systematically greater than values given by Fernie (1968). Following Fernie's considerations we can establish the period-radius relation with the division on the fundamental and first overtone pulsators, Figure 2. From the investigated

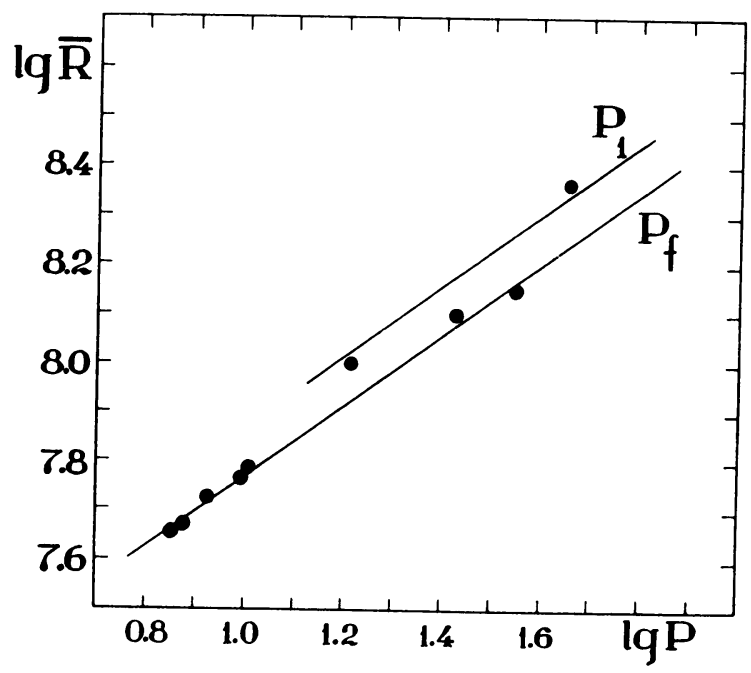

Fig. 2. Radius-period relations for fundamental and first overtone pulsators.

stars only X Cyg and SV Vul can be regarded as pulsating in the first overtone. For fundamental pulsators we have the relation:

$$
\log \bar{R}=0.904 \log P+6.88 \text {. }
$$

From the separation of two lines we can estimate the ratio of the periods $P_{1} / P_{f}$ as about 0.7 .

The values of $R_{\max }$ have been applied in the formula (1) in order to calculate the relative fluxes for Cepheids under consideration. As the absolute magnitudes the values $\left\langle M_{v}\right\rangle_{\text {int }}$ given by Fernie and Hube (1968) have been accepted, Table I. We can expect that for a homogeneous group of stars the fluxes depend chiefly on the absolute magnitude and on the spectral type. To remove the second dependence a correction for the differences of spectral types $\Delta \mathrm{Sp}$ have been used:

$$
\Delta \log F_{v}=-0.05 \Delta \mathrm{Sp},
$$




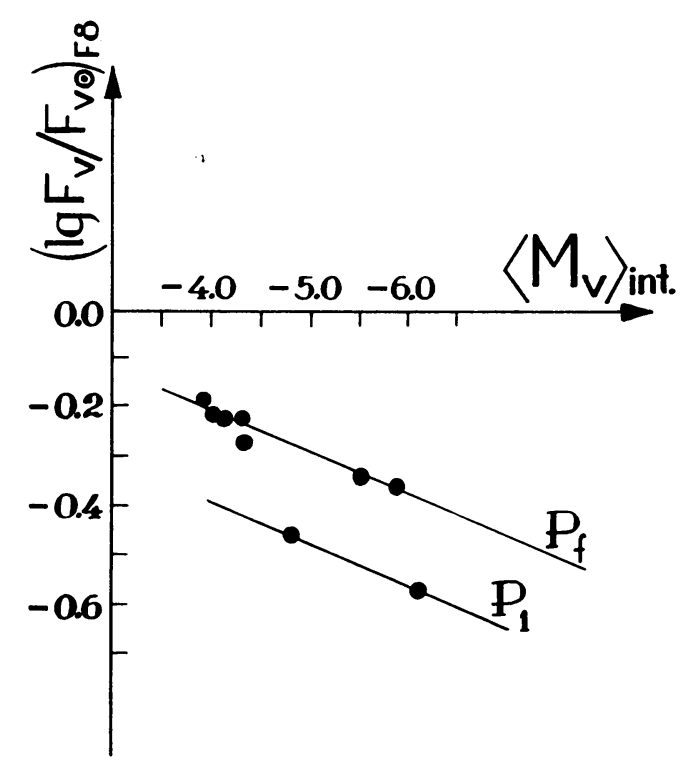

Fig. 3. Relative fluxes-absolute magnitudes relations for fundamental and first overtone pulsators.

where $\Delta \mathrm{Sp}$ is expressed in the tenth of spectral class. This formula results from the relation $(B-V)$-surface brightness given by Parsons and Bouw (1971) and from the relation $(B-V)$-spectral type. In this way we got the fluxes reduced to the mean spectral type F8. The dependance of these values on the absolute magnitudes is shown in Figure 3. Again we can observe two relations. For 7 stars recognized as fundamental pulsators we have

$$
\log F_{v} / F_{v \odot}=0.0822\left\langle M_{v}\right\rangle_{\text {int }}+0.12 .
$$

For the first overtone pulsators the values of $\log F_{v} / F_{v \odot}$ are smaller by 0.15 . These fluxes concern the state when the star in the greatest extension reaches the mean magnitude.

\section{References}

Fernie, J. D.: 1968, Astrophys. J. 151, 197.

Fernie, J. D. and Hube, J. O.: 1967, Publ. Astron. Soc. Pacific 79, 95.

Fernie, J. D. and Hube, J. O.: 1968, Astron. J. 73, 492.

Latyshev, I. N.: 1964, Astron. Zh. 41, 644.

Opolski, A. and Krawiecka, J.: 1965, Contr. Wroclaw Astron. Obs. No. 11.

Parsons, S. B. and Bouw, G. D.: 1971, Monthly Notices Roy. Astron. Soc. $152,133$. 